\title{
O-GlcNAcylation in health and neurodegenerative diseases
}

\author{
Byeong Eun Lee ${ }^{1}$, Pann-Ghill Suh ${ }^{1,2}$ and Jae-Ick Kim (D) ${ }^{1 凶}$ \\ (c) The Author(s) 2021
}

O-GlcNAcylation is a posttranslational modification that adds O-linked $\beta$ - $N$-acetylglucosamine (O-GlcNAc) to serine or threonine residues of many proteins. This protein modification interacts with key cellular pathways involved in transcription, translation, and proteostasis. Although ubiquitous throughout the body, O-GIcNAc is particularly abundant in the brain, and various proteins commonly found at synapses are O-GIcNAcylated. Recent studies have demonstrated that the modulation of O-GIcNAc in the brain alters synaptic and neuronal functions. Furthermore, altered brain O-GlcNAcylation is associated with either the etiology or pathology of numerous neurodegenerative diseases, while the manipulation of O-GlcNAc exerts neuroprotective effects against these diseases. Although the detailed molecular mechanisms underlying the functional roles of O-GlcNAcylation in the brain remain unclear, O-GlcNAcylation is critical for regulating diverse neural functions, and its levels change during normal and pathological aging. In this review, we will highlight the functional importance of O-GlcNAcylation in the brain and neurodegenerative diseases.

Experimental \& Molecular Medicine (2021) 53:1674-1682; https://doi.org/10.1038/s12276-021-00709-5

\section{INTRODUCTION}

O-GlcNAcylation, which attaches O-linked $\beta$ - $N$-acetylglucosamine (O-GlcNAc) moieties to either serine or threonine residues of intracellular proteins, is a posttranslational modification that critically regulates essential cellular functions ${ }^{1-7}$. Notably, this modification is catalyzed by only two enzymes, O-GlcNAc transferase (OGT) and O-GlcNAcase (OGA). OGT catalyzes the addition of O-GICNAc to the hydroxyl groups of serine/threonine residues of nucleocytoplasmic proteins, whereas OGA removes the modification from proteins (Fig. 1) 1,3,4,8,9. O-GlcNAcylation occurs in various cellular locations, such as the nucleus, cytosol, and cellular organelles, including mitochondria, the cytoskeleton, and the endoplasmic reticulum ${ }^{10}$. O-GIcNAcylation by OGT utilizes uridine diphosphate $\mathrm{N}$-acetylglucosamine (UDP-GlcNAc) as a precursor molecule, which is synthesized by the hexosamine biosynthesis pathway (HBP) that is pivotal for the cellular metabolism of amino acids, lipids, and nucleotides in the cell (Fig. 1) ${ }^{1}$. In the HBP, glucose is first phosphorylated by hexokinase (HK) and converted to glucose-6-phosphate (G-6P). Next, G-6P is transformed into fructose-6-phosphate (F-6P) by phosphoglucose isomerase (GPI). Then, glutamine:fructose-6-phosphate aminotransferase (GFAT), a rate-limiting enzyme of HBP, catalyzes F-6P and glutamine into glucosamine-6-phosphate (GlcN-6P). With acetyl-CoA, GlcN-6P is further catalyzed by glucosaminephosphate $N$-acetyltransferase (GNA1) and turns into $N$-acetylglucosamine-6-phosphate (GlcNAc-6P). Further isomerization by GlcNAc phosphomutase (PGM3) produces $N$-acetylglucosamine1-phosphate (GlcNAc-1P). Finally, with uridine triphosphate (UTP), UDP-GICNAc, and pyrophosphate (PPi) are produced by UDPGlcNAc pyrophosphorylase (UAP1) (Fig. 1) ${ }^{2,11}$. Notably, since O-GlcNAcylation occurs on serine/threonine residues of proteins, the O-GlcNAcylation site can also be phosphorylated, and there could be extensive crosstalk between O-GlcNAcylation and phosphorylation $^{3,8,12}$. Hence, it is conceivable that O-GlcNAcylation, through its competitive interplay with phosphorylation, could critically affect a variety of cellular signaling pathways by dynamically modulating protein activities ${ }^{8,13}$.

Mounting evidence has shown that O-GlcNAcylation regulates essential cellular processes such as gene expression, signal transduction, cell cycle, nutrient sensing, protein homeostasis, and cellular responses to diverse stress conditions (Fig. 1) ${ }^{13-18}$. In addition, altered and abnormal O-GlcNAcylation is frequently observed in multiple disease conditions, including cancer, cardiac hypertrophy, and type II diabetes ${ }^{19-23}$, supporting the notion that O-GlcNAcylation is implicated not only in maintaining normal cellular functions but also in the pathological processes of human diseases. Notably, among bodily organs, $\mathrm{O}-$ GlcNAcylation is the most abundant in the brain ${ }^{10}$. Furthermore, two related enzymes, OGT and OGA, show high expression and activity in the brain ${ }^{24,25}$. These findings strengthen the emerging notion that O-GIcNAcylation plays an important role in various neural functions. Therefore, in this review, we will focus on the importance of O-GlcNAcylation in neural functions and neurodegenerative diseases.

\section{O-GLCNACYLATION IN THE BRAIN}

The physiological importance of O-GlcNAcylation in the brain is reflected by the fact that both OGT and OGA are indispensable for neuronal survival. Genetic deletion of OGT caused embryonic stem cell lethality, while deletion of OGA led to perinatal lethality $^{26-28}$. In addition, although it is still not clear how these changes occur, neuron-specific elimination of either OGT or OGA results in severe structural abnormalities in the nervous system, which seems to be caused either by neurodevelopmental defects or premature neurodegeneration ${ }^{29-32}$. More importantly, recent

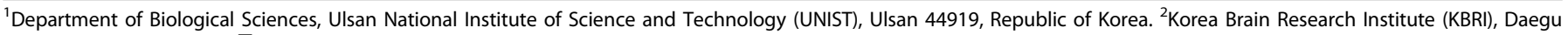
41062, Republic of Korea. ${ }^{凶}$ email: jikim220@unist.ac.kr 


\section{Hexoamine biosynthetic pathway}

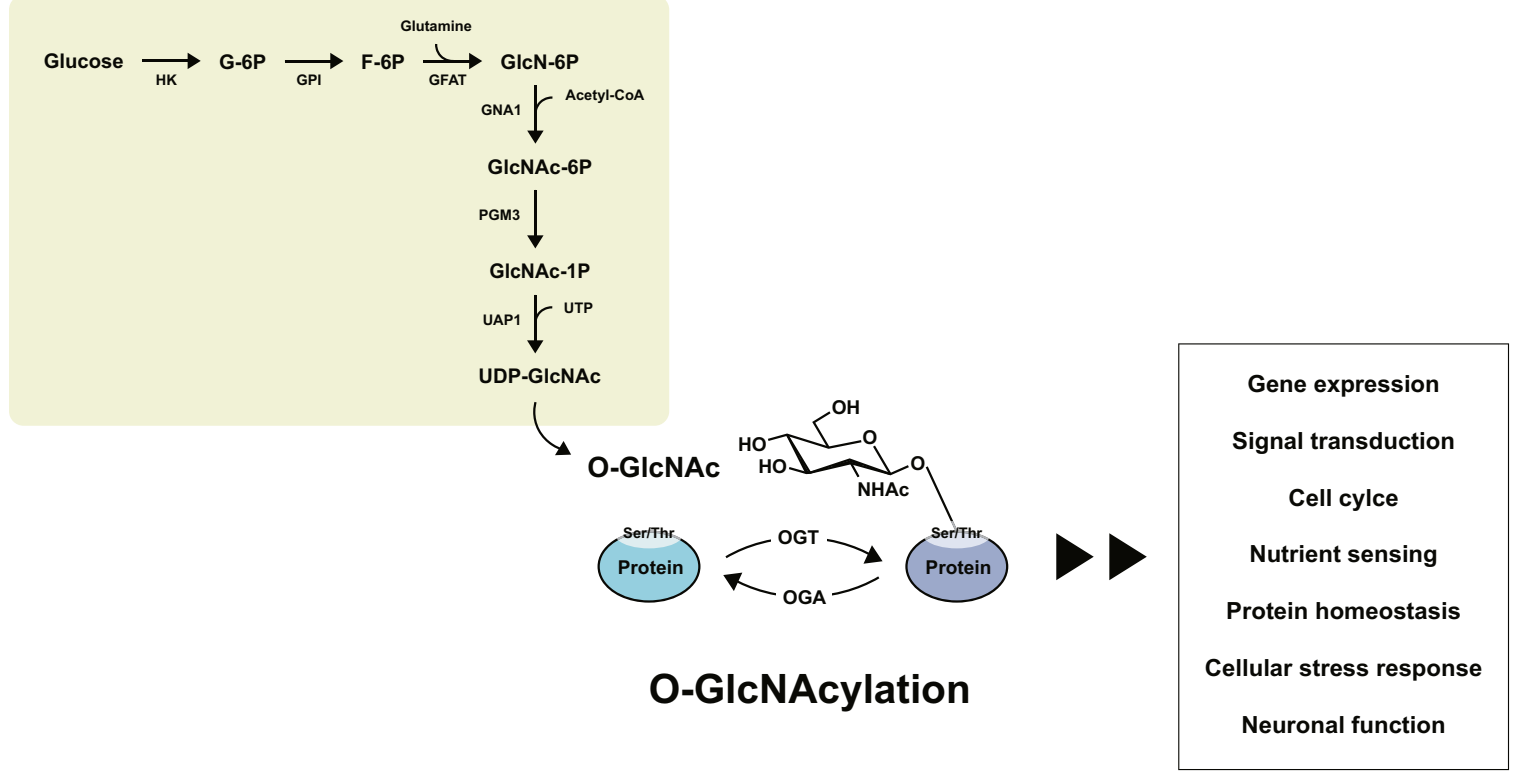

Fig. 1 O-GIcNAcylation and its regulation of various cellular processes. O-GlcNAcylation is a posttranslational modification that attaches O-GICNAc moieties to serine or threonine residues of cellular proteins. O-GlcNAcylation can regulate important cellular processes such as gene expression, signal transduction, cell cycle, nutrient sensing, protein homeostasis, cellular stress response, and neuronal function.

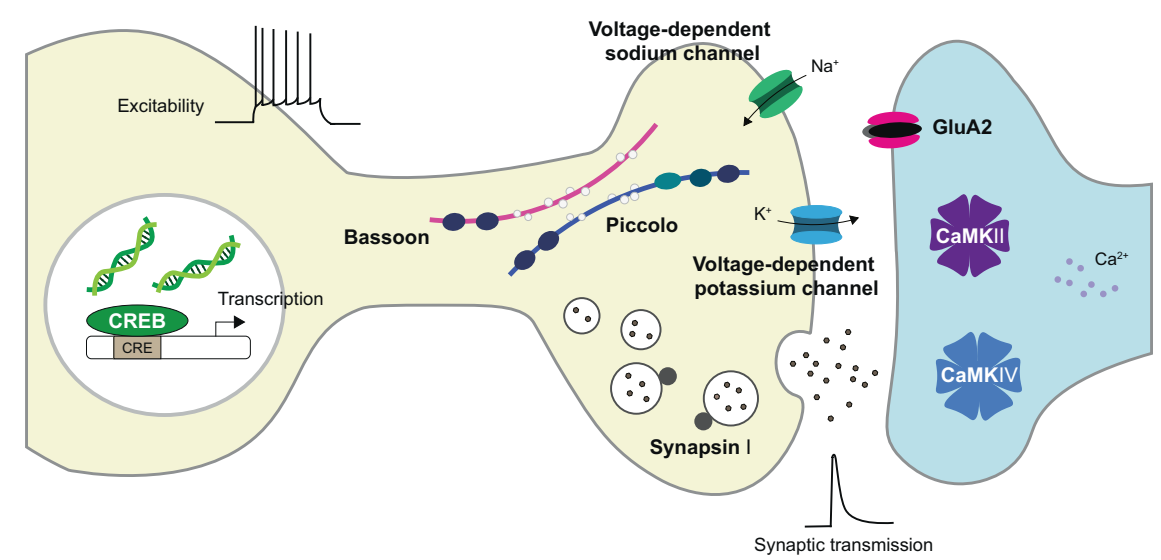

Fig. 2 Neuronal and synaptic proteins modified by O-GIcNAcylation. Various neuronal and synaptic proteins, including bassoon, piccolo, synapsin I, GluA2, CaMKII, CaMKIV, and CREB, are modified by O-GIcNAcylation. These modifications critically regulate neuronal and synaptic properties.

studies are beginning to show that O-GlcNAcylation is crucial not only for neuronal survival but also for neuronal and synaptic function in the mature brain. O-GlcNAcylation in the brain is highly enriched, especially at synapses. OGA and OGT are significantly active at functional synapses, leading to extensive O-GICNAc modification of proteins in nerve terminals ${ }^{33}$. Moreover, many synaptic and neuronal proteins important for structure and function, such as bassoon, synapsin I, piccolo, synaptopodin, GluA2, calcium/calmodulin-dependent kinases II (CaMKII), CaMKIV, and cyclic adenosine monophosphate (AMP)response element-binding protein (CREB), are modified by O-GlcNAcylation (Fig. 2) ${ }^{15,34-38}$. O-GlcNAcylation of these proteins was found to critically regulate the functional properties of neurons in multiple neural circuits.

When O-GlcNAc was acutely increased by an OGA inhibitor, thiamet-G (TMG), neuronal excitability, and excitatory synaptic transmission of hippocampal neurons were suppressed. A transient enhancement of O-GlcNAcylation elevated the amplitude of the voltage-dependent potassium channel current and the expression of hyperpolarization-activated cyclic nucleotide-gated (HCN) channels and reduced the size of voltage-gated sodium channels ${ }^{39}$. This suppression of neuronal excitability by O-GlcNAcylation in multiple cation channels clearly demonstrates the ability of O-GlcNAc modification to regulate neuronal function. Agouti-related protein (AgRP)-expressing neurons in the hypothalamus showed enriched OGT expression, and selective knockout of OGT in these AgRP neurons inhibited their spontaneous firing activity due to the defective action of the voltage-gated potassium channel Kcnq3 (Kv7.3) by loss of O-GlcNAc modification in this potassium channel ${ }^{40}$. Forebrain excitatory neuron-specific deletion of OGT by using mice expressing a tamoxifen-inducible Cre recombinase under the control of the CaMKIla promoter also promoted hyperphagia-dependent obesity by attenuating excitatory input to the paraventricular nucleus (PVN) neurons in the hypothalamus ${ }^{41}$. Consistent with this finding, OGT is enriched in the postsynaptic density of excitatory synapses, and removal of OGT from cultured cortical neurons markedly 
1676

reduced the number of mature excitatory synapses by regulating the synaptic expression of a-amino-3-hydroxy-5-methyl-4-isoxazolepropionic acid (AMPA) receptors ${ }^{42}$. Dopaminergic neuron-specific elimination of OGT caused early loss of axonal arborization and premature neurodegeneration, whereas enhancement of O-GlcNAcylation by knocking out OGA in dopamine neurons did not negatively affect neuronal structures. Interestingly, these dopamine neuron-specific OGA knockout mice exhibited enhanced dopamine release at dopamine terminals in the striatum, suggesting the potential role of O-GlcNAcylation in modulating the synaptic release machinery ${ }^{32}$. These studies collectively indicate that neuronal O-GlcNAcylation significantly regulates not only the survival or maintenance of neurons but also the physiological functions of synapses and neurons in the brain. Given the diverse roles played by O-GlcNAcylation in key cellular processes, much needs to be identified with respect to O-GlcNAcylated neuronal proteins, their O-GlcNAcylation sites, and the brain-specific signaling mechanisms selectively affected by O-GlcNAc modification.

\section{O-GLCNACYLATION AND NEURODEGENERATIVE DISEASES Aging}

Considering the critical role of O-GlcNAcylation in regulating synaptic and neuronal functions, it is reasonable to assume that the alteration of O-GIcNAc levels during aging could exert deleterious effects on numerous neural functions in the brain. Several reports have shown that the abundance of O-GlcNAcylation changes depending on aging conditions ${ }^{43-48}$. In the hippocampus of aged mice, both OGT expression and the level of O-GlcNAcylation were decreased compared with those in young mice, and this loss of O-GlcNAcylation by age impaired cognitive function ${ }^{44}$. Age-dependent loss of O-GlcNAcylation in neural stem cells (NCS) is correlated with reduced neurogenesis and increased gliogenesis in the hippocampus ${ }^{47}$. Notably, decreased O-GlcNAcylation in the NSCs of young mice by conditional knockout of OGT led to a reduction in adult neurogenesis and impaired hippocampal-dependent learning and memory ${ }^{47}$. The relationship between O-GlcNAcylation and aging was also found in invertebrate organisms such as Caenorhabditis elegans. The oga-1 C. elegans mutant showed altered gene expression compared with the wild-type, and these differentially expressed genes were largely associated with the aging pathway and lifespan ${ }^{49}$. Moreover, modulating O-GlcNAC levels by manipulating HBP revealed a protective effect on the aging and lifespan of $C$. elegans. Lifespan was significantly increased in the gfat-1 gain-of-function mutants, and supplementation with GlcNAc was sufficient to extend the lifespan by alleviating proteotoxicity and improving protein quality control ${ }^{50}$. Supplementation with glucosamine (GlcN) also resulted in an extended lifespan in both nematodes and aged mice ${ }^{51}$. Collectively, these findings identify a critical role of O-GlcNAcylation and related pathways in aging and lifespan.

Moreover, according to recent studies, alterations of O-GlcNAcylation in various neuronal proteins are found in agingrelated neurodegenerative diseases, including Alzheimer's disease (AD) and Parkinson's disease (PD) ${ }^{52,53}$. Numerous proteins associated with neurogenerative diseases appear to be O-GlcNAcylated, and their O-GIcNAcylation status can change the progression of disease pathology (Table 1$)^{8,54,55}$. Tau protein, one of the bestknown hallmarks of $A D$, is O-GlcNAcylated in the brain ${ }^{56-58}$. It has been shown that the O-GlcNAcylation of tau protein mitigates pathological aggregates of tau and, as a consequence, ameliorates cellular toxicity caused by aggregated tau ${ }^{59}$. Thus, elucidation of the alterations of O-GlcNAcylation in neurodegenerative diseases not only provides a more comprehensive view of the role of O-GlcNAcylation in the brain but also is vital to the identification of novel therapeutic targets and therapies against currently untreatable neurodegenerative diseases such as AD.

\section{Alzheimer's disease}

Alzheimer's disease (AD) is the most common neurodegenerative disease and is characterized by progressive mental deterioration. Symptoms start with mild memory deficits and mood changes and progress to severe cognitive impairment and difficulties in swallowing and urination, eventually resulting in death ${ }^{60}$. Recently, researchers found that the O-GlcNAc level is 22 to $50 \%$ lower in $A D$ patients than in healthy controls ${ }^{57,61-63}$. As such, it is plausible that O-GlcNAcylation is associated with the pathogenesis and/or progression of AD pathology. In addition to amyloid-beta, tau protein has been shown to play a major role in the pathology of $A D^{64,65}$. Tau is a microtubule-associated protein that modulates the stability of the neuronal cytoskeleton, and the activity of tau protein, like many other proteins, is highly regulated by phosphorylation. In the AD brain, tau is abnormally hyperphosphorylated and then accumulates. This accumulation of hyperphosphorylated tau eventually forms neurofibrillary tangles (NFTs) that induce significant cellular toxicity ${ }^{64,65}$. Importantly, tau protein is extensively O-GIcNAcylated ${ }^{56,57}$, and several studies have revealed that O-GlcNAcylation of tau can effectively reduce tauopathy. Treatment with an OGA inhibitor, thiamet-G, in PC-12 cells expressing tau protein substantially diminished tau phosphorylation at Ser396 and Thr231, the initial priming phosphorylation sites of pathological tau, and this result was also replicated in the rat brain after thiamet-G treatment in vivo ${ }^{66}$. Among $A D$ mouse models, the JNPL3 mouse is a transgenic mouse model that overexpresses mutant human P301L tau. This mouse develops neurofibrillary tangles, gliosis, and neurodegeneration in an age-dependent manner due to tau hyperphosphorylation $^{67}$. When the JNPL3 mice were treated with thiamet-G, the phosphorylated tau at Ser202 and Thr205 was remarkably reduced in the brain. Moreover, this decrease in pathological tau phosphorylation led to the attenuation of NFT formation and neuronal death, suggesting a neuroprotective effect of O-GIcNAcylation in the AD brain ${ }^{59}$.

Amyloid- $\beta(A \beta)$ plaques are another hallmark of $A D$. $A \beta$ is a peptide whose toxic oligomeric form is widely found in the brains of $A D$ patients ${ }^{68}$. The 5xFAD mouse is a well-studied AD model overexpressing human mutant $A \beta$ precursor protein and shows severe amyloid pathology, including elevated extracellular $A \beta$ plaques. When 5xFAD mice were treated with an OGA inhibitor, the number of $A \beta$ plaques was remarkably reduced compared to that in vehicle-treated mice ${ }^{69}$. This reduction in $A \beta$ plaques was caused by the attenuated activity of $\gamma$-secretase, a critical enzyme for $A \beta$ generation. Furthermore, the activity of $\gamma$-secretase was attenuated through O-GlcNAcylation at the Ser708 residue of nicastrin (NCT), which is an essential component of the $\gamma$-secretase complex and acts as a substrate receptor ${ }^{69,70}$. From the above, it is apparent that in addition to the regulation of NFT formation, O-GlcNAcylation might be critical for the modulation of $\gamma$-secretase activity and the resulting $A \beta$ production. The reactivity of microglia and astrocytes was also significantly reduced by OGA inhibition, and this reduction in amyloid pathology resulted in the recovery of impaired memory function in $5 \times F A D$ mice ${ }^{69}$. Genetically increased O-GICNAc levels due to insufficient OGA also recovered impaired cognitive function in 5xFAD mice $\left(5 \times F A D ; \mathrm{OGA}^{\mathrm{f} /++}\right)^{63}$. The amount of $A \beta$ in $5 \times F A D ; O G A^{f / /}$ mice was significantly reduced compared with that in $5 \times F A D$ mice, resulting in attenuation of neuronal death. This alleviation of pathology in $5 \times \mathrm{FAD} ; \mathrm{OGA}^{\mathrm{fl} /+}$ mice, in turn, markedly restored cognitive function ${ }^{63}$. Given that the elevation of O-GlcNAcylation does not cause any harmful effects on the structure and function of neurons ${ }^{32,59}$, it is conceivable that increasing O-GlcNAcylation would have a neuroprotective effect against $A D$ with minimal side effects. Recently, selective and smallmolecule OGA inhibitors such as MK-8719 and ASN120290 (previously known as ASN-561) have entered early clinical trials for the treatment of progressive supranuclear palsy $(P S P)^{71}$. Moreover, LY3372689, another OGA inhibitor, is being developed 

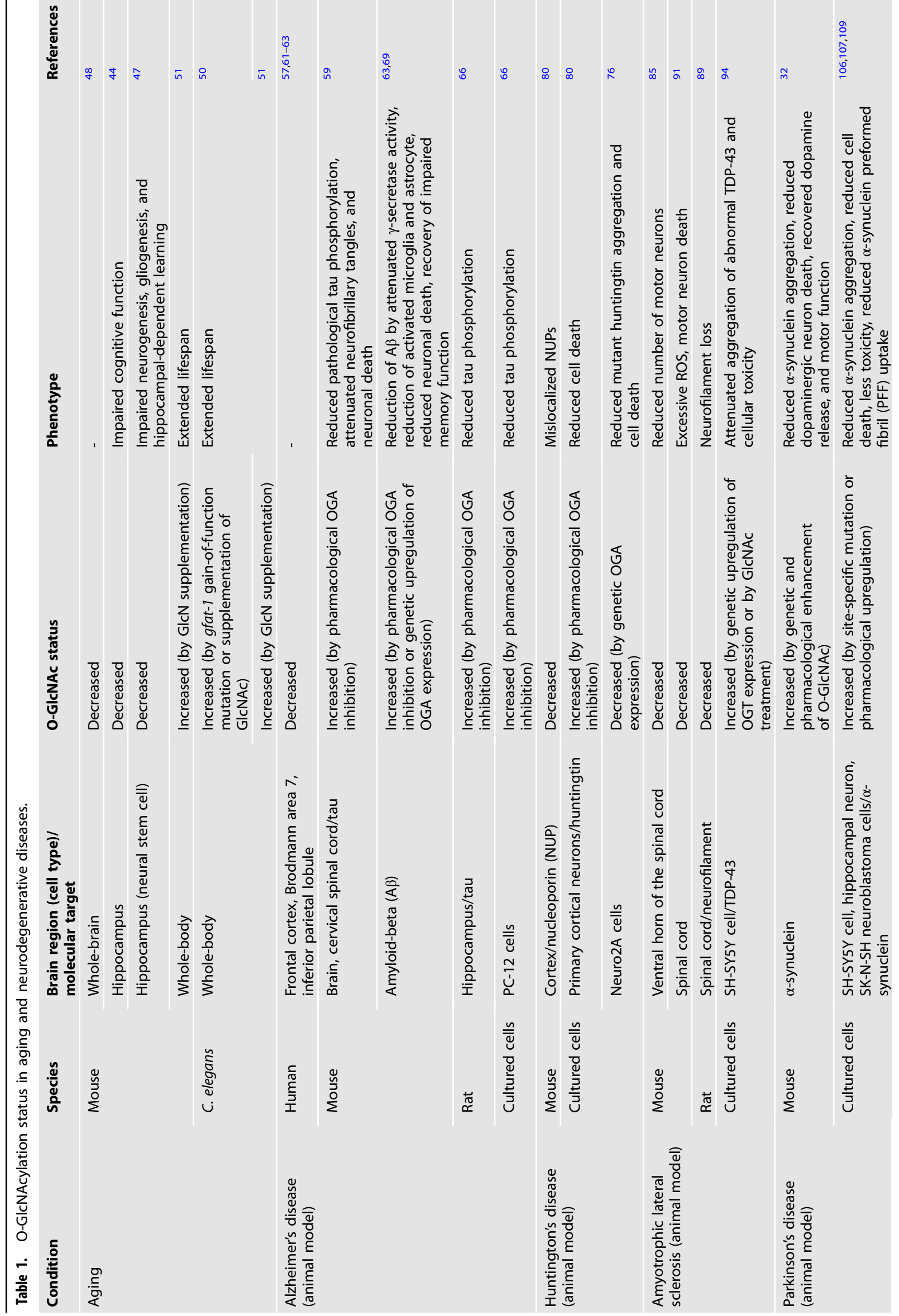
1678

for the treatment of tauopathies, including AD. Hopefully, the results from these clinical trials may be utilized to justify whether brain-permeable OGA inhibitors can be pursued for further clinical development against AD.

\section{Huntington's disease}

Huntington's disease (HD) is a rare, inherited neurodegenerative disease that is characterized by progressive neurodegeneration, motor impairment, and cognitive dysfunction ${ }^{72,73}$. The main cause of $\mathrm{HD}$ is a mutation of the huntingtin $(H T T)$ gene coding the huntingtin protein ( $\mathrm{HTT}$ ). Originally, the $H T T$ gene contains up to 34 glutamine-coding (CAG) repeats. By mutations, this repeat expands to polyglutamine repeats and causes misfolding of the huntingtin protein ${ }^{74}$. This abnormally folded protein is prone to aggregate, readily forming oligomers. These oligomers are seeds for larger inclusions and pathogenic mutant huntingtin (mHTT) fibrils $^{74}$. Functionally, these $\mathrm{mHTT}$ fibrils and aggregates interrupt synaptic transmission, mitochondrial axonal transport, and gene transcription $^{74}$. To date, the relationship between O-GlcNAcylation and HD has been unclear. Interestingly, however, HTT protein and huntingtin-interacting protein 1-related protein (HIP1R) appear to be O-GlcNAc-modified, indicating that HTT protein could be functionally regulated by O-GIcNAcylation ${ }^{32,75}$. When the HTT protein construct with polyglutamine repeats was coexpressed with OGA in Neuro2A cells (murine neuroblastoma cell line), the number of transfected cells with mutant huntingtin aggregates was significantly reduced. Coexpression of the OGA constructs also led to a significant reduction in cell death mediated by $\mathrm{mHTT}$ aggregation ${ }^{76}$. In contrast to the findings in cases of $A D$, these results support the possibility that lowering O-GIcNAcylation levels can mitigate the aggregation and cellular toxicity of the $\mathrm{mHTT}$ protein.

In HD mouse models, distortion of the nuclear membrane and disrupted nucleocytoplasmic transport were also observed ${ }^{77}$. Nucleoporin (NUP) is a component of the nuclear pore complex that is required for selective transport across the nuclear pore. Notably, mislocalization of this NUP was found in HD mouse models. R6/2 and zQ175 mice are HD mouse models expressing human $\mathrm{mHTT}$ with expanded CAG repeats ${ }^{78,79}$. In R6/2 mice that develop progressive motor and cognitive deficits as early as 6-8 weeks of age, NUP62, which is important for controlling nuclear pore permeability and selective transport, formed intranuclear inclusions and was colocalized with $\mathrm{mHTT}$ aggregates ${ }^{80}$. Consistent with this finding, intracellular inclusions formed by NUP88 were found and colocalized with $\mathrm{mHTT}$ aggregates in zQ175 mice ${ }^{80}$. This NUP mislocalization and pathology were also observed in postmortem human brains from HD patients ${ }^{80}$. Intriguingly, NUP is heavily O-GlcNAcylated, and its O-GlcNAcylation appears to be critical for nuclear pore integrity and selective filtration ${ }^{81}$. Strengthening this result, there was a reduction in O-GlcNAcylation in ZQ175 mice, and treatment of primary cortical neurons transfected with $\mathrm{HTT} 82 \mathrm{Q}$ with an OGA inhibitor markedly reduced cell death, indicating that elevation of O-GlcNAc levels could restore nucleocytoplasmic trafficking ${ }^{80}$. On the basis of these observations, NUP mislocalization in HD pathology is likely linked to altered levels of O-GlcNAcylation. Thus, it is increasingly evident that O-GlcNAcylation may be related to HD pathology by modulating $\mathrm{mHTT}$ aggregation and nucleocytoplasmic transport. Further elucidation of the exact role of O-GlcNAcylation in HD pathology will lead to a better understanding of the molecular etiology and pathophysiology of HD.

\section{Amyotrophic lateral sclerosis}

Amyotrophic lateral sclerosis (ALS), also known as Lou Gehrig's disease, is a neurodegenerative motor neuron disease that causes the degeneration of both lower and upper motor neurons in the motor cortex, brain stem nuclei, and anterior horn of the spinal cord $^{82-84}$. This loss of motor neurons results in focal limbic muscle weakness and progresses to respiratory failure, limiting survival to 2-4 years after disease onset ${ }^{82,83}$. Nevertheless, the exact mechanism of motor neuron degeneration remains incompletely understood. Several factors, such as genetic mutation, abnormal neurofilament function, oxidative stress, and inflammation, are considered the main causes of $\mathrm{ALS}^{84}$. In ALS, $90 \%$ of cases are classified as sporadic ALS, and the remaining $10 \%$ of cases are familial ALS with dominantly inherited autosomal mutations in SOD1 (superoxide dismutase 1), TDP-43 (TAR DNA-binding protein 43), FUS (fused in sarcoma/translated in liposarcoma), and C9orf72 (chromosome 9 open reading frame 72$)^{83}$. According to recent studies of ALS, O-GICNAcylation can play a neuroprotective role against ALS pathology. It was reported that the abundance of O-GlcNAcylation in mutant SOD1-overexpressing mice was significantly reduced in the motor neurons of the spinal cord compared with that of wild-type mice ${ }^{85}$. Interestingly, hyperphosphorylated neurofilaments (NFs) and their aggregation are observed in ALS pathology ${ }^{86,87}$, and this neurofilament is modified by O-GIcNAc ${ }^{32,88}$. Consistent with these findings, O-GlcNAcylation of neurofilaments remarkably decreased in the ALS rat model overexpressing mutant SOD1, potentially indicating the disruption of O-GICNAcylation in ALS pathology ${ }^{89}$. Considering the crosstalk with phosphorylation, O-GlcNAcylation may suppress excessive phosphorylation of neurofilaments and concomitantly alleviate ALS pathology.

In addition, the elevation of oxidative stress is one of the primary factors leading to the degeneration of motor neurons in $\mathrm{ALS}^{83,84}$. Nonselenocysteine-containing phospholipid hydroperoxide glutathione peroxidase (NPGPx) is an oxidative stress sensor and transmitter that modulates protein activity by shuffling disulfide bonds ${ }^{90}$. Notably, NPGPx knockout mice showed ALS-like phenotypes such as paralysis, denervation of neuromuscular junctions, and motor neuron loss. Importantly, these mice displayed dysregulation of O-GIcNAc levels and ROS accumulation, consequently causing motor neuron death. However, this loss of motor neurons was recovered by elevating O-GICNAC levels with an OGA inhibitor ${ }^{91}$. These results suggest that O-GIcNAcylation can modulate ALS pathology by reducing ROS accumulation. TDP-43 protein pathology is also a hallmark of ALS. TDP-43 is an RNA/DNA-binding protein and acts as a regulator of transcription, mRNA stability, and transport ${ }^{92}$. The excessive accumulation of TDP-43 in the cytoplasm produces inclusion bodies, which induce cellular toxicity by abnormal protein/RNA interactions ${ }^{84,93}$. A recent study reported that the O-GIcNAcylation of endogenous TDP-43 was found in human SH-SY5Y neuroblastoma cells, and mass spectrometry analysis further detected O-GlcNAcylation at the T199 and T233 sites of TDP-4394. As in the aforementioned cases, coexpression of OGT with an ALS-linked mutant of TDP-43 significantly suppressed abnormal TDP-43 aggregation and related cellular toxicity ${ }^{94}$. Together, these studies indicate a critical role of O-GIcNAcylation in modulating ALS pathology by inhibiting excessive phosphorylation and protein aggregation and reducing ROS accumulation. The molecular mechanisms underlying the role of O-GlcNAcylation in ALS pathology need to be further elucidated.

\section{Parkinson's disease}

Parkinson's disease (PD) is the second most common neurodegenerative disease after AD. PD patients generally develop motor symptoms, including rigidity, bradykinesia, and postural instability ${ }^{95-98}$. However, alongside these motor symptoms, they also experience nonmotor symptoms such as sleep disruption, depression, and even cognitive decline years before the diagnosis of $\mathrm{PD}^{98,99}$. The loss of dopaminergic neurons in the midbrain and the functional disruption of the basal ganglia circuitry are the key features of PD pathophysiology ${ }^{96,100}$. Among pathological markers of the disease, Lewy bodies (LBs), composed of an abnormal aggregation of proteins, are a well-known pathological hallmark of PD that can induce neuronal toxicity, and a-synuclein is the 
major component of LB inclusions ${ }^{101,102}$. a-Synuclein, contributing a large proportion of PD pathology, is expressed abundantly in the brain and is primarily located in presynaptic terminals and synaptic vesicles ${ }^{102,103}$. Although the function of a-synuclein is still unclear, a-synuclein is known to regulate the mobility, release, and maintenance of synaptic vesicles ${ }^{102,103}$. As with other aggregation-prone proteins, a-synuclein phosphorylation can induce abnormal aggregation, and this aggregation, in turn, is capable of significantly disturbing cellular structure and function, which eventually causes neuronal death ${ }^{104}$. Notably, a-synuclein is also O-GlcNAc modified ${ }^{104,105}$. When a-synuclein was O-GlcNAcylated in a site-specific manner, the abnormal aggregation of a-synuclein by phosphorylation was markedly diminished, resulting in alleviated neurotoxicity in cultured neurons ${ }^{106-108}$. In these experiments, a-synuclein containing O-GlcNAc at T72 did not form any aggregates or oligomers and inhibited phosphorylation at S87 and S129, which has been found to be closely related to a-synuclein aggregation in PD pathology. In addition, treatment with O-GIcNAcylated a-synuclein in cultured neurons reduced neuronal death ${ }^{106}$. Despite its variable impact depending on the specific O-GlcNAcylation sites, O-GlcNAcylated asynuclein largely showed an inhibitory effect on aggregation.
The O-GlcNAcylation of a-synuclein at T72, T75, and T81 remarkably inhibited or attenuated a-synuclein aggregation compared to that of unmodified a-synuclein, and triple O-GlcNAcylation of a-synuclein at all of these sites completely blocked aggregation ${ }^{107}$. In a similar vein, the elevation of O-GICNAc in neuroblastoma cells by OGA inhibition reduced the uptake of a-synuclein preformed fibrils (PFFs) without interrupting the normal endocytosis capacity ${ }^{109}$. Taken together, these findings strongly indicate that O-GIcNAcylation of a-synuclein can attenuate pathological aggregation of a-synuclein and a-synuclein-mediated neurotoxicity in vitro.

Recent findings from our group also demonstrated that O-GlcNAcylation plays an important role in the function, survival, and degeneration of dopamine neurons in vivo in a mouse model of $\mathrm{PD}^{32}$. In our study, dopaminergic neuron-specific OGT knockout mice exhibited a significant loss of dopamine neurons in the midbrain and premature death at $\sim 8$ to 15 weeks of age. In contrast, selective enhancement of O-GlcNAcylation in dopamine neurons did not negatively impact neuronal structures or survival. Rather, it facilitated synaptic transmission at dopamine synapses in mice with OGA conditional knockout ${ }^{32}$. These results indicate that O-GlcNAcylation is essential for neuronal survival and function in

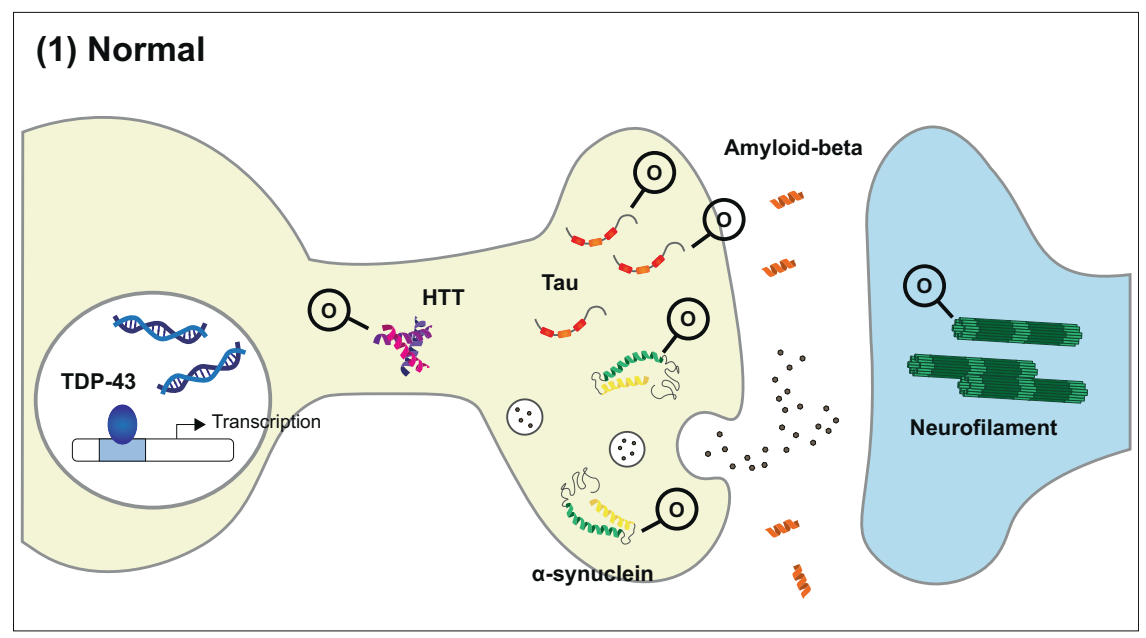

(2) Neurodegenerative disease (altered O-GIcNAcylation)
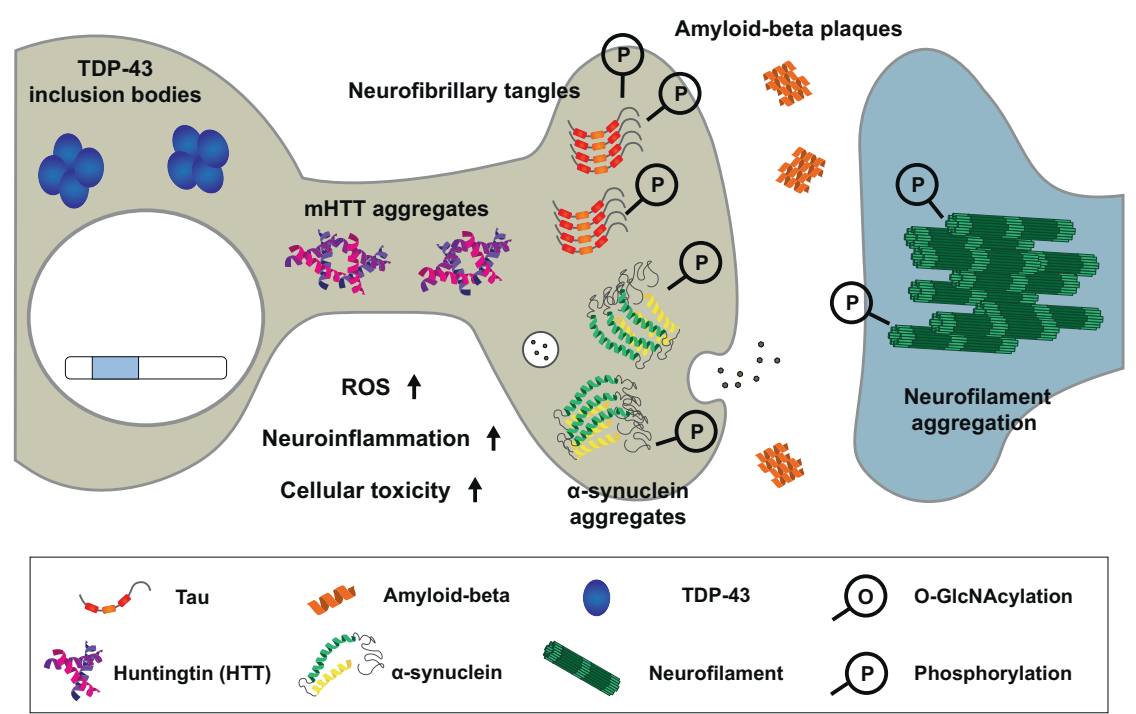

Fig. 3 O-GlcNAcylation and its neuroprotective role in neurons. Key etiological proteins of neurodegenerative diseases, such as tau, $\alpha$-synuclein, HTT, and neurofilament, can be directly O-GIcNAcylated, and proteins, including amyloid-beta and TDP-43, are indirectly affected by O-GlcNAcylation. In neurodegenerative diseases, altered O-GlcNAcylation is detected, and this abnormal O-GlcNAc status and subsequent excessive phosphorylation can cause pathological protein aggregation, resulting in cellular toxicity in neurons. 
dopamine neurons. More interestingly, when we generated a mouse model of PD by overexpressing mutant $a$-synuclein in the midbrain of mice with OGA conditional knockout, the elevated O-GlcNAcylation dramatically alleviated PD pathology in dopamine neurons, including abnormal aggregation of a-synuclein and neuronal death. As a functional consequence of these changes, impaired dopamine release and motor behaviors observed in PD model mice were significantly recovered by enhanced O-GlcNAcylation ${ }^{32}$. Together, the elevation of O-GlcNAc levels in vivo can alleviate PD pathology and related physiological symptoms, possibly by inhibiting the pathological aggregation of a-synuclein. Given that both genetic and pharmacological elevation of O-GlcNAc did not cause any harmful effects on brain function and general health in mice ${ }^{32}$, it may be clinically promising to develop PD therapies focusing on O-GlcNAcylation. More research should be conducted to identify molecular targets of O-GlcNAcylation in dopamine neurons and their pathophysiological roles in PD.

\section{CONCLUSION AND PERSPECTIVES}

O-GlcNAcylation critically contributes to various cellular processes, including transcription, translation, signaling cascades, and protein homeostasis, in a multitude of cell types, and more than 5000 human proteins have been identified as O-GlcNAcylated proteins thus far ${ }^{10}$. Notably, recent studies on O-GlcNAcylation have begun to emphasize the importance of O-GlcNAcylation in the central nervous system. There is undoubtedly evidence indicating a pivotal role of O-GlcNAcylation in the brain. First, O-GICNAC modification is most abundant in the brain among various organs. Second, numerous proteins enriched in and important for functional synapses are O-GlcNAcylated. Most importantly, accumulating studies have demonstrated that genetic or pharmacological manipulation of O-GlcNAcylation remarkably alters neuronal and synaptic functions in the brain. Moreover, the critical role of O-GlcNAcylation is not limited to the normal, healthy brain. Several proteins known as key etiological factors in neurodegenerative diseases, including tau, amyloid-beta precursor protein (APP), a-synuclein, and $\mathrm{HTT}$, are O-GlcNAcylated. In addition, the status of O-GlcNAcylation in these proteins is associated with many pathological conditions in neurodegenerative diseases. In experimental studies, altering O-GlcNAc levels in the brain or cultured neurons reduces aberrant protein aggregation, which is a common hallmark of neurodegenerative diseases. Moreover, the manipulation of O-GlcNAcylation (mostly enhancement) in neurodegenerative disease-related proteins mitigates neurotoxicity, functional impairment, and neuronal death, strongly suggesting the neuroprotective effect of O-GIcNAcylation (Fig. 3). It is important to note that, in most cases, either genetic or pharmacological manipulation of O-GlcNAc levels does not lead to any deleterious effects on the structure and function of neurons in the brain; instead, it specifically suppresses neurodegenerative disease-related pathology in animal models. Thus, it is worth pursuing O-GlcNAcylation as a therapeutic target for many neurodegenerative diseases, such as $A D$ and PD.

However, caution should be exercised because O-GlcNAcylation is a dynamic modification that reversibly interacts with other posttranslational modifications, including phosphorylation, ubiquitylation, acetylation, and methylation, and causes complicated modulation of diverse proteins in the cytoplasm and nucleus ${ }^{110}$. Depending on the specific brain regions, cell types, and pathological conditions, the effect of O-GIcNAc modification in the brain can be variable and may even be harmful in some cases. In addition, another important issue researchers should address when investigating O-GlcNAcylation is the reliable detection of O-GlcNAcylated proteins, which is still challenging for several reasons. First, since the donor substrate of O-GIcNAc modification, UDP-GICNAc, is produced by the sequential integration of nutrients, including glucose, amino acids, fatty acids, and nucleotides, via HBP,
O-GlcNAcylation is highly sensitive to the metabolic states of cells in living animals $2,3,12$. Second, even though O-GlcNAcylation itself is abundant in the brain, each individual O-GICNAc-modified protein is generally present in low abundance ${ }^{33}$. Finally, O-GlcNAcylation is labile and easily lost from peptides during the process of dissociation ${ }^{111}$. Fortunately, however, recent progress in proteomics and new emerging strategies in the detection of O-GlcNAcylation have enabled us to overcome these aforementioned difficulties/ issues ${ }^{112}$. In addition, the development of single-cell isolation and analysis can further provide detailed profiling of individual cellspecific responses, ranging from gene expression to proteomics ${ }^{113}$. Undoubtedly, this technology may greatly encourage the interpretation of neuron type-specific and protein-specific effects of O-GlcNAcylation in the near future.

In this review, we briefly described recent progress demonstrating the neuroprotective effects of O-GIcNAcylation in a variety of neurodegenerative diseases. Our understanding of O-GlcNAcylation in the brain continues to evolve at a rapid pace, but its practical application for therapeutic goals is still in its infancy. Moving forward, the utilization of advanced new technologies will allow us to dissect the molecular alterations by O-GIcNAcylation that determine disease-specific pathology and its alleviation in neurodegenerative diseases.

\section{REFERENCES}

1. Zachara, N. E. \& Hart, G. W. Cell signaling, the essential role of O-GlcNAc! Biochim. Biophys. Acta 1761, 599-617 (2006).

2. Hardiville, S. \& Hart, G. W. Nutrient regulation of signaling, transcription, and cell physiology by O-GlcNAcylation. Cell Metab. 20, 208-213 (2014).

3. Bond, M. R. \& Hanover, J. A. A little sugar goes a long way: the cell biology of O-GIcNAc. J. Cell Biol. 208, 869-880 (2015).

4. Bond, M. R. \& Hanover, J. A. O-GlcNAc cycling: a link between metabolism and chronic disease. Annu. Rev. Nutr. 33, 205-229 (2013).

5. Lewis, B. A. \& Hanover, J. A. O-GlcNAc and the epigenetic regulation of gene expression. J. Biol. Chem. 289, 34440-34448 (2014).

6. Torres, C. R. \& Hart, G. W. Topography and polypeptide distribution of terminal $\mathrm{N}$-acetylglucosamine residues on the surfaces of intact lymphocytes. Evidence for O-linked GIcNAc. J. Biol. Chem. 259, 3308-3317 (1984).

7. Hanover, J. A., Cohen, C. K., Willingham, M. C. \& Park, M. K. O-linked N-acetylglucosamine is attached to proteins of the nuclear pore. Evidence for cytoplasmic and nucleoplasmic glycoproteins. J. Biol. Chem. 262, 9887-9894 (1987).

8. Hart, G. W., Slawson, C., Ramirez-Correa, G. \& Lagerlof, O. Cross talk between O-GlcNAcylation and phosphorylation: roles in signaling, transcription, and chronic disease. Annu. Rev. Biochem. 80, 825-858 (2011).

9. Banerjee, P. S., Hart, G. W. \& Cho, J. W. Chemical approaches to study O-GlcNAcylation. Chem. Soc. Rev. 42, 4345-4357 (2013).

10. Wulff-Fuentes, E. et al. The human O-GlcNAcome database and meta-analysis. Sci. Data. 8, 25 (2021).

11. Marshall, S., Bacote, V. \& Traxinger, R. R. Discovery of a metabolic pathway mediating glucose-induced desensitization of the glucose transport system. Role of hexosamine biosynthesis in the induction of insulin resistance. J. Biol. Chem. 266, 4706-4712 (1991).

12. Butkinaree, C., Park, K. \& Hart, G. W. O-linked beta-N-acetylglucosamine (OGlcNAc): extensive crosstalk with phosphorylation to regulate signaling and transcription in response to nutrients and stress. Biochim. Biophys. Acta 1800, 96-106 (2010).

13. Chatham, J. C., Zhang, J. \& Wende, A. R. Role of O-linked N-acetylglucosamine protein modification in cellular (Patho)physiology. Physiol. Rev. 101, 427-493 (2021).

14. Housley, M. P. et al. O-GlcNAc regulates FoxO activation in response to glucose. J. Biol. Chem. 283, 16283-16292 (2008).

15. Erickson, J. R. et al. Diabetic hyperglycaemia activates CaMKII and arrhythmias by O-linked glycosylation. Nature 502, 372-376 (2013).

16. Lu, S. et al. Hyperglycemia acutely increases cytosolic reactive oxygen species via O-linked GlcNAcylation and CaMKII activation in mouse ventricular myocytes. Circ. Res. 126, e80-e96 (2020).

17. Jiang, M. et al. Elevated O-GlcNAcylation promotes gastric cancer cells proliferation by modulating cell cycle related proteins and ERK $1 / 2$ signaling. Oncotarget 7, 61390-61402 (2016).

18. Han, C. et al. O-GIcNAcylation of SIRT1 enhances its deacetylase activity and promotes cytoprotection under stress. Nat. Commun. 8, 1491 (2017). 
19. Slawson, C. \& Hart, G. W. O-GlcNAc signalling: implications for cancer cell biology. Nat. Rev. Cancer 11, 678-684 (2011).

20. Dassanayaka, S. \& Jones, S. P. O-GIcNAc and the cardiovascular system. Pharmacol. Ther. 142, 62-71 (2014).

21. Slawson, C., Copeland, R. J. \& Hart, G. W. O-GIcNAc signaling: a metabolic link between diabetes and cancer? Trends Biochem. Sci. 35, 547-555 (2010).

22. Ferrer, C. M. et al. O-GIcNAcylation regulates cancer metabolism and survival stress signaling via regulation of the HIF-1 pathway. Mol. Cell. 54, 820-831 (2014).

23. Gelinas, R. et al. AMPK activation counteracts cardiac hypertrophy by reducing O-GlcNAcylation. Nat. Commun. 9, 374 (2018).

24. Gao, Y., Wells, L., Comer, F. I., Parker, G. J. \& Hart, G. W. Dynamic O-glycosylation of nuclear and cytosolic proteins: cloning and characterization of a neutral, cytosolic beta-N-acetylglucosaminidase from human brain. J. Biol. Chem. 276, 9838-9845 (2001)

25. Okuyama, R. \& Marshall, S. UDP-N-acetylglucosaminyl transferase (OGT) in brain tissue: temperature sensitivity and subcellular distribution of cytosolic and nuclear enzyme. J. Neurochem 86, 1271-1280 (2003).

26. O'Donnell, N., Zachara, N. E., Hart, G. W. \& Marth, J. D. Ogt-dependent Xchromosome-linked protein glycosylation is a requisite modification in somatic cell function and embryo viability. Mol. Cell. Biol. 24, 1680-1690 (2004).

27. Keembiyehetty, C. et al. Conditional knock-out reveals a requirement for O-linked N-Acetylglucosaminase (O-GlcNAcase) in metabolic homeostasis. J. Biol. Chem. 290, 7097-7113 (2015).

28. Yang, Y. R. et al. O-GlcNAcase is essential for embryonic development and maintenance of genomic stability. Aging Cell 11, 439-448 (2012).

29. Olivier-Van Stichelen, S., Wang, P., Comly, M., Love, D. C. \& Hanover, J. A. Nutrient-driven O-linked $\mathrm{N}$-acetylglucosamine (O-GIcNAc) cycling impacts neurodevelopmental timing and metabolism. J. Biol. Chem. 292, 6076-6085 (2017).

30. Su, C. \& Schwarz, T. L. O-GlcNAc transferase is essential for sensory neuron survival and maintenance. J. Neurosci. 37, 2125-2136 (2017).

31. Wang, A. C., Jensen, E. H., Rexach, J. E., Vinters, H. V. \& Hsieh-Wilson, L. C. Loss of O-GIcNAc glycosylation in forebrain excitatory neurons induces neurodegeneration. Proc. Natl Acad. Sci. USA 113, 15120-15125 (2016).

32. Lee, B. E. et al. O-GlcNAcylation regulates dopamine neuron function, survival and degeneration in Parkinson disease. Brain 143, 3699-3716 (2020).

33. Cole, R. N. \& Hart, G. W. Cytosolic O-glycosylation is abundant in nerve terminals. J. Neurochem. 79, 1080-1089 (2001).

34. Vosseller, $\mathrm{K}$. et al. O-linked $\mathrm{N}$-acetylglucosamine proteomics of postsynaptic density preparations using lectin weak affinity chromatography and mass spectrometry. Mol. Cell Proteom. 5, 923-934 (2006).

35. Khidekel, N., Ficarro, S. B., Peters, E. C. \& Hsieh-Wilson, L. C. Exploring the O-GlcNAc proteome: direct identification of O-GlcNAc-modified proteins from the brain. Proc. Natl Acad. Sci. USA 101, 13132-13137 (2004).

36. Taylor, E. W. et al. O-GIcNAcylation of AMPA receptor GluA2 is associated with a novel form of long-term depression at hippocampal synapses. J. Neurosci. 34, 10-21 (2014).

37. Dias, W. B., Cheung, W. D., Wang, Z. \& Hart, G. W. Regulation of calcium/calmodulin-dependent kinase IV by O-GIcNAc modification. J. Biol. Chem. 284, 21327-21337 (2009)

38. Rexach, J. E. et al. Dynamic O-GICNAc modification regulates CREB-mediated gene expression and memory formation. Nat. Chem. Biol. 8, 253-261 (2012).

39. Hwang, H. \& Rhim, H. Acutely elevated O-GlcNAcylation suppresses hippocampal activity by modulating both intrinsic and synaptic excitability factors. Sci. Rep. 9, 7287 (2019).

40. Ruan, H. B. et al. O-GlcNAc transferase enables AgRP neurons to suppress browning of white fat. Cell 159, 306-317 (2014).

41. Lagerlof, O. et al. The nutrient sensor OGT in PVN neurons regulates feeding. Science 351, 1293-1296 (2016)

42. Lagerlof, O., Hart, G. W. \& Huganir, R. L. O-GlcNAc transferase regulates excitatory synapse maturity. Proc. Natl Acad. Sci. USA 114, 1684-1689 (2017).

43. Banerjee, P. S., Lagerlof, O. \& Hart, G. W. Roles of O-GIcNAc in chronic diseases of aging. Mol. Asp. Med. 51, 1-15 (2016).

44. Wheatley, E. G. et al. Neuronal O-GlcNAcylation improves cognitive function in the aged mouse brain. Curr. Biol. 29, 3359-3369 e3354 (2019)

45. Rex-Mathes, M. et al. O-GlcNAc expression in developing and ageing mouse brain. Biochimie 83, 583-590 (2001).

46. Fulop, N. et al. Aging leads to increased levels of protein O-linked N-acetylglucosamine in heart, aorta, brain and skeletal muscle in Brown-Norway rats. Biogerontology 9, 139 (2008).

47. White, C. W. 3rd et al. Age-related loss of neural stem cell O-GlcNAc promotes a glial fate switch through STAT3 activation. Proc. Natl Acad. Sci. USA 117, 22214-22224 (2020).

48. Wang, Z. et al. Increasing O-GlcNAcylation is neuroprotective in young and aged brains after ischemic stroke. Exp. Neurol. 339, 113646 (2021).
49. Love, D. C. et al. Dynamic O-GlcNAc cycling at promoters of Caenorhabditis elegans genes regulating longevity, stress, and immunity. Proc. Natl Acad. Sci. USA 107, 7413-7418 (2010).

50. Denzel, M. S. et al. Hexosamine pathway metabolites enhance protein quality control and prolong life. Cell 156, 1167-1178 (2014).

51. Weimer, S. et al. D-Glucosamine supplementation extends life span of nematodes and of ageing mice. Nat. Commun. 5, 3563 (2014).

52. Lazarus, B. D., Love, D. C. \& Hanover, J. A. O-GlcNAc cycling: implications for neurodegenerative disorders. Int. J. Biochem. Cell Biol. 41, 2134-2146 (2009).

53. Ma, X., Li, H., He, Y. \& Hao, J. The emerging link between O-GlcNAcylation and neurological disorders. Cell Mol. Life Sci. 74, 3667-3686 (2017).

54. Wani, W. Y., Chatham, J. C., Darley-Usmar, V., McMahon, L. L. \& Zhang, J. O-GIcNAcylation and neurodegeneration. Brain Res. Bull. 133, 80-87 (2017).

55. Ryan, P. et al. O-GICNAc modification protects against protein misfolding and aggregation in neurodegenerative disease. ACS Chem. Neurosci. 10, 2209-2221 (2019).

56. Arnold, C. S. et al. The microtubule-associated protein tau is extensively modified with O-linked N-acetylglucosamine. J. Biol. Chem. 271, 28741-28744 (1996).

57. Liu, F., Iqbal, K., Grundke-lqbal, I., Hart, G. W. \& Gong, C. X. O-GlcNAcylation regulates phosphorylation of tau: a mechanism involved in Alzheimer's disease. Proc. Natl Acad. Sci. USA 101, 10804-10809 (2004).

58. Morris, M. et al. Tau post-translational modifications in wild-type and human amyloid precursor protein transgenic mice. Nat. Neurosci. 18, 1183-1189 (2015).

59. Yuzwa, S. A. et al. Increasing O-GlcNAc slows neurodegeneration and stabilizes tau against aggregation. Nat. Chem. Biol. 8, 393-399 (2012).

60. Breijyeh, Z. \& Karaman, R. Comprehensive review on Alzheimer's disease: causes and treatment. Molecules 25, 5789 (2020).

61. Liu, F. et al. Reduced O-GlcNAcylation links lower brain glucose metabolism and tau pathology in Alzheimer's disease. Brain 132, 1820-1832 (2009).

62. Balana, A. T. et al. O-GIcNAc modification of small heat shock proteins enhances their anti-amyloid chaperone activity. Nat. Chem. 13, 441-450 (2021).

63. Park, J. et al. O-GlcNAcylation ameliorates the pathological manifestations of Alzheimer's disease by inhibiting necroptosis. Sci. Adv. 7, eabd3207 (2021).

64. Iqbal, K. et al. Tau pathology in Alzheimer disease and other tauopathies. Biochim. Biophys. Acta 1739, 198-210 (2005).

65. Kopeikina, K. J., Hyman, B. T. \& Spires-Jones, T. L. Soluble forms of tau are toxic in Alzheimer's disease. Transl. Neurosci. 3, 223-233 (2012).

66. Yuzwa, S. A. et al. A potent mechanism-inspired O-GlcNAcase inhibitor that blocks phosphorylation of tau in vivo. Nat. Chem. Biol. 4, 483-490 (2008).

67. Lewis, J. et al. Neurofibrillary tangles, amyotrophy and progressive motor disturbance in mice expressing mutant (P301L) tau protein. Nat. Genet. 25, 402-405 (2000).

68. Busche, M. A. \& Hyman, B. T. Synergy between amyloid-beta and tau in Alzheimer's disease. Nat. Neurosci. 23, 1183-1193 (2020)

69. Kim, C. et al. O-linked beta-N-acetylglucosaminidase inhibitor attenuates betaamyloid plaque and rescues memory impairment. Neurobiol. Aging 34, 275-285 (2013).

70. Shah, S. et al. Nicastrin functions as a gamma-secretase-substrate receptor. Cell 122, 435-447 (2005).

71. Medina, M. An overview on the clinical development of Tau-based therapeutics. Int. J. Mol. Sci. 19, 1160 (2018).

72. McColgan, P. \& Tabrizi, S. J. Huntington's disease: a clinical review. Eur. J. Neurol. 25, 24-34 (2018).

73. Walker, F. O. Huntington's disease. Lancet 369, 218-228 (2007).

74. Tabrizi, S. J., Flower, M. D., Ross, C. A. \& Wild, E. J. Huntington disease: new insights into molecular pathogenesis and therapeutic opportunities. Nat. Rev. Neurol. 16, 529-546 (2020)

75. Stephanie, O. V.-S. The human O-GIcNAcome database. figshare. https://figshare. com/articles/dataset/The_human_O-GlcNAcome_database/12443495 (2020).

76. Kumar, A. et al. Decreased O-linked GlcNAcylation protects from cytotoxicity mediated by huntingtin exon1 protein fragment. J. Biol. Chem. 289, 13543-13553 (2014).

77. Gasset-Rosa, F. et al. Polyglutamine-expanded Huntingtin exacerbates agerelated disruption of nuclear integrity and nucleocytoplasmic transport. Neuron 94, 48-57 e44 (2017).

78. Tang, B. et al. Gene expression profiling of R6/2 transgenic mice with different CAG repeat lengths reveals genes associated with disease onset and progression in Huntington's disease. Neurobiol. Dis. 42, 459-467 (2011).

79. Menalled, L. B. et al. Comprehensive behavioral and molecular characterization of a new knock-in mouse model of Huntington's disease: zQ175. PLOS ONE 7, e49838 (2012).

80. Grima, J. C. et al. Mutant Huntingtin disrupts the nuclear pore complex. Neuron 94, 93-107 e106 (2017).

81. Zhu, Y. et al. Post-translational O-GlcNAcylation is essential for nuclear pore integrity and maintenance of the pore selectivity filter. J. Mol. Cell Biol. 8, 2-16 (2016). 
82. Masrori, P. \& Van Damme, P. Amyotrophic lateral sclerosis: a clinical review. Eur. J. Neurol. 27, 1918-1929 (2020).

83. Mejzini, R. et al. ALS genetics, mechanisms, and therapeutics: where are we now? Front. Neurosci. 13, 1310 (2019).

84. Morgan, S. \& Orrell, R. W. Pathogenesis of amyotrophic lateral sclerosis. Br. Med. Bull. 119, 87-98 (2016).

85. Shan, X., Vocadlo, D. J. \& Krieger, C. Reduced protein O-glycosylation in the nervous system of the mutant SOD1 transgenic mouse model of amyotrophic lateral sclerosis. Neurosci. Lett. 516, 296-301 (2012).

86. Xiao, S., McLean, J. \& Robertson, J. Neuronal intermediate filaments and ALS: a new look at an old question. Biochim. Biophys. Acta 1762, 1001-1012 (2006).

87. Lobsiger, C. S., Garcia, M. L., Ward, C. M. \& Cleveland, D. W. Altered axonal architecture by removal of the heavily phosphorylated neurofilament tail domains strongly slows superoxide dismutase 1 mutant-mediated ALS. Proc. Natl Acad. Sci. USA 102, 10351-10356 (2005).

88. Dong, D. L., Xu, Z. S., Hart, G. W. \& Cleveland, D. W. Cytoplasmic O-GlcNAC modification of the head domain and the KSP repeat motif of the neurofilament protein neurofilament-H. J. Biol. Chem. 271, 20845-20852 (1996).

89. Ludemann, N. et al. O-glycosylation of the tail domain of neurofilament protein $M$ in human neurons and in spinal cord tissue of a rat model of amyotrophic lateral sclerosis (ALS). J. Biol. Chem. 280, 31648-31658 (2005).

90. Chen, Y. I., Wei, P. C., Hsu, J. L., Su, F. Y. \& Lee, W. H. NPGPx (GPx7): a novel oxidative stress sensor/transmitter with multiple roles in redox homeostasis. Am. J. Transl. Res. 8, 1626-1640 (2016).

91. Hsieh, Y. L. et al. NPGPX-mediated adaptation to oxidative stress protects motor neurons from degeneration in aging by directly modulating O-GlcNAcase. Cell Rep. 29, 2134-2143 e2137 (2019).

92. Cohen, T. J., Lee, V. M. \& Trojanowski, J. Q. TDP-43 functions and pathogenic mechanisms implicated in TDP-43 proteinopathies. Trends Mol. Med. 17, 659-667 (2011).

93. Saberi, S., Stauffer, J. E., Schulte, D. J. \& Ravits, J. Neuropathology of amyotrophic lateral sclerosis and its variants. Neurol. Clin. 33, 855-876 (2015).

94. Zhao, M. J. et al. O-GlcNAcylation of TDP-43 suppresses proteinopathies and promotes TDP-43's mRNA splicing activity. EMBO Rep. 22, e51649 (2021).

95. Kalia, L. V. \& Lang, A. E. Parkinson's disease. Lancet 386, 896-912 (2015).

96. Dauer, W. \& Przedborski, S. Parkinson's disease: mechanisms and models. Neuron 39, 889-909 (2003).

97. Davie, C. A. A review of Parkinson's disease. Br. Med. Bull. 86, 109-127 (2008).

98. Dexter, D. T. \& Jenner, P. Parkinson disease: from pathology to molecular disease mechanisms. Free Radic. Biol. Med. 62, 132-144 (2013).

99. Schapira, A. H. V., Chaudhuri, K. R. \& Jenner, P. Non-motor features of Parkinson disease. Nat. Rev. Neurosci. 18, 435-450 (2017).

100. Smith, Y., Wichmann, T., Factor, S. A. \& DeLong, M. R. Parkinson's disease therapeutics: new developments and challenges since the introduction of levodopa. Neuropsychopharmacol. 37, 213-246 (2012).

101. Spillantini, M. G. et al. Alpha-synuclein in Lewy bodies. Nature $\mathbf{3 8 8}, \mathbf{8 3 9 - 8 4 0}$ (1997).

102. Kim, W. S., Kagedal, K. \& Halliday, G. M. Alpha-synuclein biology in Lewy body diseases. Alzheimers Res. Ther. 6, 73 (2014).

103. Bras, I. C. \& Outeiro, T. F. Alpha-synuclein: mechanisms of release and pathology progression in synucleinopathies. Cells 10, 375 (2021).

104. Zhang, J., Li, X. \& Li, J. D. The roles of post-translational modifications on alphasynuclein in the pathogenesis of Parkinson's diseases. Front. Neurosci. 13, 381 (2019).

105. Wang, Z. et al. Enrichment and site mapping of O-linked $\mathrm{N}$-acetylglucosamine by a combination of chemical/enzymatic tagging, photochemical cleavage, and electron transfer dissociation mass spectrometry. Mol. Cell Proteom. 9, 153-160 (2010).

106. Marotta, N. P. et al. O-GlcNAc modification blocks the aggregation and toxicity of the protein alpha-synuclein associated with Parkinson's disease. Nat. Chem. 7, 913-920 (2015).
107. Levine, P. M. et al. alpha-Synuclein O-GIcNAcylation alters aggregation and toxicity, revealing certain residues as potential inhibitors of Parkinson's disease. Proc. Natl Acad. Sci. USA 116, 1511-1519 (2019).

108. Galesic, A. et al. Comparison of $\mathrm{N}$-acetyl-glucosamine to other monosaccharides reveals structural differences for the inhibition of alpha-synuclein aggregation. ACS Chem. Biol. 16, 14-19 (2021).

109. Tavassoly, O., Yue, J. \& Vocadlo, D. J. Pharmacological inhibition and knockdown of O-GIcNAcase reduces cellular internalization of alpha-synuclein preformed fibrils. FEBS J. 288, 452-470 (2021).

110. Yang, X. \& Qian, K. Protein O-GlcNAcylation: emerging mechanisms and functions. Nat. Rev. Mol. Cell Biol. 18, 452-465 (2017).

111. Cecioni, S. \& Vocadlo, D. J. Tools for probing and perturbing O-GlcNAc in cells and in vivo. Curr. Opin. Chem. Biol. 17, 719-728 (2013).

112. $\mathrm{Xu}, \mathrm{S}$., Sun, F., Tong, M. \& Wu, R. MS-based proteomics for comprehensive investigation of protein O-GlcNAcylation. Mol. Omics 17, 186-196 (2021).

113. Hu, P., Zhang, W., Xin, H. \& Deng, G. Single cell isolation and analysis. Front. Cell Dev. Biol. 4, 116 (2016).

\section{ACKNOWLEDGEMENTS}

This research was supported by the Basic Science Research Program through the National Research Foundation of Korea (NRF) funded by the Ministry of Science and ICT (2020R1A2C1005492 and 2021R1A4A1031644 to J.-I.K.). This research was also supported by the Asan Foundation Biomedical Research Fellowship (B.E.L.).

\section{AUTHOR CONTRIBUTIONS}

Conceptualization, B.E.L. and J.-I.K.; original draft preparation, B.E.L. and J.-I.K.; review and editing, P.-G.S. and J.-I.K.; supervision, J.-I.K. All authors have read and agreed to the current version of the manuscript.

\section{COMPETING INTERESTS}

The authors declare no competing interests.

\section{ADDITIONAL INFORMATION}

Correspondence and requests for materials should be addressed to Jae-lck Kim.

Reprints and permission information is available at http://www.nature.com/ reprints

Publisher's note Springer Nature remains neutral with regard to jurisdictional claims in published maps and institutional affiliations.

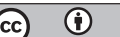

Open Access This article is licensed under a Creative Commons Attribution 4.0 International License, which permits use, sharing, adaptation, distribution and reproduction in any medium or format, as long as you give appropriate credit to the original author(s) and the source, provide a link to the Creative Commons license, and indicate if changes were made. The images or other third party material in this article are included in the article's Creative Commons license, unless indicated otherwise in a credit line to the material. If material is not included in the article's Creative Commons license and your intended use is not permitted by statutory regulation or exceeds the permitted use, you will need to obtain permission directly from the copyright holder. To view a copy of this license, visit http://creativecommons. org/licenses/by/4.0/.

(c) The Author(s) 2021 